\title{
Analysis and Calculation of Threshold Pressure Gradient Based on Capillary Bundle
}

\author{
Shuangshuang Ren, ${ }^{1,2}$ Fei Shen $\mathbb{D}^{1,2}$ Shenglai Yang, ${ }^{3}$ Xiangyang Zhang, ${ }^{1,2}$ Hongwu Luo, ${ }^{1,2}$ \\ and Chaomin Feng ${ }^{1,2}$ \\ ${ }^{1}$ Northwest Branch of China Petroleum Exploration and Development Research Institute, Lanzhou 730000, Gansu, China \\ ${ }^{2}$ Key Laboratory of Internet of Things, CNPC, Lanzhou 730000, Gansu, China \\ ${ }^{3}$ College of Petroleum Engineering, China University of Petroleum, Beijing 102249, China
}

Correspondence should be addressed to Fei Shen; swfswfswf2005@126.com

Received 25 February 2021; Revised 2 April 2021; Accepted 6 May 2021; Published 27 May 2021

Academic Editor: Jianguo Wang

Copyright (c) 2021 Shuangshuang Ren et al. This is an open access article distributed under the Creative Commons Attribution License, which permits unrestricted use, distribution, and reproduction in any medium, provided the original work is properly cited.

Oilfield water injection is one of the important means to supplement energy to the formation and enhance oil recovery in the process of oilfield development. The level of water injection technology determines the effect of oilfield development and also determines the length of oilfield development life. Research on seepage law of water injection development in low-permeability reservoir is the basis and important technical means of low-permeability reservoir development, and the key point of seepage law is to analyze the starting pressure gradient law. In previous studies, either static test or dynamic experimental value is used, so the error of pseudo starting pressure gradient derived from experimental value is too large, which makes people expand the starting pressure value in low-permeability reservoir in practical engineering application, and the starting pressure gradient obtained from laboratory test cannot be applied in actual reservoir. To accurately calculate the threshold pressure gradient for low-permeability reservoirs, the threshold permeability is proposed through the study of the seepage law and laboratory experiments. It is recognized that the threshold pressure gradient and the threshold permeability had been changing during the seepage. Through steady-state "flow rate-pressure difference" displacement experiment, with natural cores from a low-permeability reservoir, based on a capillary bundle model, the method for calculating the gradient is innovatively proposed. The experimental data show that the whole low-permeability seepage flow is nonlinear, divided into three stages according to the physical stages with obvious changes. Through processing and analyzing of the experimental results, first, it is showed that both threshold pressure gradient and threshold permeability increase with the rise of flow rate and the increasing amplitude is gradually decreasing. Second, the study proposes the permeability is the main controlling reason of the threshold pressure gradient, and the flow velocity is an important reason. Third, we obtain the formulas of the minimum threshold pressure gradient, the threshold pressure gradient, and the corresponding threshold permeability of different cores and the power function relationship between the threshold pressure gradient and the core permeability is obtained. And further, the one-dimensional experimental results are applied to the radial fluid flow, and the recognition that the threshold pressure gradient decreases with increasing distance and the ratio of the threshold pressure to the total displacement pressure difference are obtained. The ratio of starting pressure to total pressure drop is about 0.5 , and the higher the permeability is, the lower the ratio is lower under 0.5 . These findings significantly help in understanding how to effectively develop low-permeability reservoir by water injection. Through the dynamic macro experiment and microcapillary bundle principle, the experiment can be divided into several sections for analysis, which can be more accurate. The minimum start-up pressure gradient can not only guide the later development of the oilfield, but also enrich the theoretical study of non-Darcy low-velocity seepage. At the same time, the law of flow velocity and start-up pressure gradient indirectly proves the boundary layer theory of the generation mechanism of start-up pressure gradient and supports and guides the effective development of various development methods of low-permeability reservoir. 


\section{Introduction}

In low-permeability reservoirs, the fluid seepage does not obey Darcy's law and therefore it is necessary to overcome the minimum threshold pressure gradient fluid to seep. The predecessors studied the threshold pressure gradient, including the theoretical analysis of the threshold pressure gradient mechanism [1], the experimental measurement of threshold pressure gradient [2-4], the numerical simulation of threshold pressure gradient $[5,6]$, and using production data to calculate threshold pressure gradient [7]. The minimum threshold pressure gradient is generally measured by the capillary equilibrium method [8], but this method takes a long time. The straight line fitting method is generally used to measure the threshold pressure gradient, but this method is related to the selection of flow velocity and pressure gradient, by which different threshold pressure gradient results are calculated by different people. Xu et al. proposed that the threshold pressure gradient is changeable [9], who used the derivation of the fitting formulae to conduct theoretical research, but the research result was not used in the processing of the experimental results.

During the development of low-permeability reservoirs, due to the existence of threshold pressure gradient, the injection-production pressure difference is large, which causes some low-permeability intervals being poorly developed. Therefore, studying the change rule of threshold pressure gradient as well as studying the relationship between the threshold pressure gradient and the reservoir physical properties has realistic guiding significance for the development of the reservoir development, well pattern deployment, and separated zone water injection, etc. [10].

The research is divided into the following parts. First, the experimental section is presented. Then, the results and discussion are put forward. Last, summary and conclusions are given.

In previous studies, either static test or dynamic experimental value is used, so the error of pseudo starting pressure gradient derived from experimental value is too large, which makes people expand the starting pressure value in low-permeability reservoir in practical engineering application, and the starting pressure gradient obtained from laboratory test cannot be applied in an actual reservoir. This paper put forward threshold permeability, set up a capillary bundle model for analyzing the change of starting pressure gradient, and experimented by the steady flow "flow-pressure difference" method. By fitting the experiment result to every three data points in the neighborhood, the threshold permeability and the threshold pressure gradient values were obtained after fitting. Finally, the threshold pressure gradient was obtained through mapping and interpolation. Further, the change law of threshold pressure gradient and threshold permeability with flow rate was analyzed. Based on this change rule, the minimum threshold pressure gradient value and threshold pressure gradient under different flow rates were extrapolated to better describe the low-permeability reservoir percolation process. It is considered that the threshold pressure gradient decreases with the distance from the center of the well, and the threshold pressure is not the product of the pressure gradient and the distance to be started.

Through the dynamic macro experiment and microcapillary bundle principle, the experiment can be divided into several sections for analysis, which can be more accurate.

The minimum start-up pressure gradient can not only guide the later development of the oilfield, but also enrich the theoretical study of non-Darcy low-velocity seepage.

1.1. The Definition of Threshold Pressure Gradient. In a lowpermeability reservoir, when the driving pressure gradient is small, the liquid cannot flow. Only when the driving pressure gradient reaches a certain value does the liquid start to flow. Now, the driving pressure gradient is called the threshold pressure gradient. The higher the threshold pressure gradient is, the more difficult it is for crude oil to flow and the poorer water absorption capacity in the oil interval. Therefore, the basic law of oil and water seepage in low-permeability reservoirs is different from that in high permeability reservoirs [5].

Predecessors have done some research on the mechanism of the threshold pressure gradient, mainly including assumptions: fluid boundary layer is abnormal in nature [11]. The fluid in seepage is composed of body fluid and boundary fluid. The boundary fluid is plastic fluid, in which the flow and pressure difference follow the Buckingham-Reiner formula [12]. From this, it can be seen that the fluid sees a nonlinear flow in the low-permeability reservoir, which is the comprehensive result of the fluid boundary layer anomaly and the behavior of the fluid in the porous medium as a plastic fluid.

Threshold pressure gradient can be divided into the minimum threshold pressure gradient, the threshold pressure gradient, and the maximum threshold pressure gradient or critical threshold pressure gradient [7]. Point $A$ in Figure 1 is the minimum threshold pressure gradient. The fluid begins to flow above the pressure gradient matching to this point, which can also be called the threshold pressure gradient. Point $B$ is the threshold pressure gradient which is the intersection of the extension line of the straight line slice and the pressure gradient axis. Point $C$ is the maximum threshold pressure gradient or critical pressure gradient. Above the pressure gradient matching to the point $C$, the fluids in the pores all join in the flow, which meets the linear flow law. $D$ is the transition point from nonlinear seepage to quasi linear seepage. The seepage of the linear $\mathrm{DE}$ is quasi linear seepage, and the seepage matching to the curve AD is nonlinear seepage.

\section{Experiment}

2.1. Conditions and Methods. The minimum threshold pressure gradient is generally measured using the capillary equilibrium method [9], but this method takes a long time. To measure the threshold pressure gradient is to use the steady-state method or the non-steady-state method to 


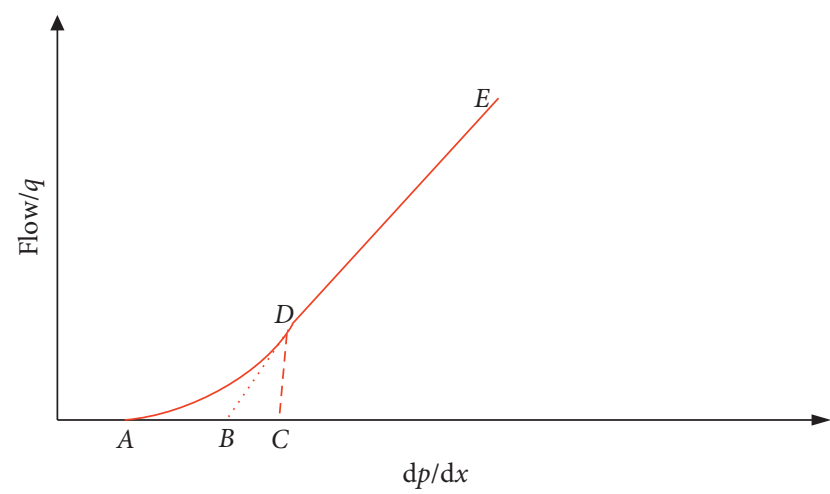

Figure 1: A typical non-Darcy flow curve.

decide the pressure difference and flow in the seepage through the indoor physical simulation experiment. And then the mathematical model and the matching processing method are used to calculate the threshold pressure gradient. However, there are some disadvantages in the seepage law described of low-permeability cores.

In the experiment, because the flow rate and pressure gradient range need to be selected and determined as a straight line segment, the threshold pressure gradients calculated by the straight line segments corresponding to different experimental points are different. Besides, no experimental standard measures the threshold pressure gradient, and the results of measurements at different flow rates and pressure gradients are different $[13,14]$.

The main experimental equipment for this study is the high-precision microflow constant-speed pump and the pressure gauge. The flow rate of the water is controlled by a computer and the upstream pressure of the core is automatically collected. The experiment temperature is $20^{\circ} \mathrm{C}$, and the core is the natural core from a low-permeability block in oilfield. The basic data are shown in Table 1. The experimental water is formulated according to the formation water composition in the study area, of which the salinity is $6813 \mathrm{mg} / \mathrm{L}$, the density is $1.008 \mathrm{~g} / \mathrm{cm}^{3}$, and the viscosity is $1.077 \mathrm{MPa}$ s.

\subsection{Procedures. The experimental steps are as follows:}

(1) Dry core sample and weigh it, and then vacuum the core sample for $24 \mathrm{~h}$. After that, keep the core sample saturated in formation water while keep vacuuming the core sample $12 \mathrm{~h}$.

(2) Keep the core sample stand for $24 \mathrm{~h}$ after saturated in formation water and then weigh the core sample.

(3) Keep the constant-speed displacement at a certain speed while keeping the confining pressure higher than the flow pressure $2.5 \mathrm{MPa}$, and record the flow rate and pressure difference after the pressure is stabilized.

(4) Increase flow rate and continue the constant-speed displacement procedure by using the increased flow rate and repeat step (3).
(5) End the experiment and process the experimental data.

\section{Results and Discussion}

\subsection{The Comprehensive Analysis of Experimental Results}

3.1.1. The Conventional Method for Determining the Threshold Pressure Gradient. General capillary equilibrium method is used to measure the minimum threshold pressure gradient [9], but this method takes longer. Quasi threshold pressure gradient on indoor physical simulation experiment of measurement is using steady or unsteady seepage differential pressure and flow rate determination, using mathematical model and matching processing methods to solve the threshold pressure gradient. There are certain problems in the theory of obtaining the low permeability start-up pressure through linear slope regression based on the experimental data of flow and pressure because the data points selected for the experiment are different and the slope of the line obtained is different, so the start-up pressure values are different $[13,14]$.

What stands out in Figure 2 (the data are shown in Table 2) is the markedly nonlinear of the relationship between the driving pressure gradient and the flow rate. With the increase of the pressure gradient, most of the roars have been opened, and the relationship between core flow and pressure gradient tends to be a straight line.

3.1.2. Analysis of Threshold Pressure Gradient Based on Capillary Bundle Model. It is assumed that the core is composed of many capillaries in parallel; the smaller the core pore throat radius, the greater the boundary layer thickness, and the bigger the threshold pressure gradient $[1,15]$. Each capillary has a radius; the same radius of capillary tube matches the same threshold pressure gradient and permeability.

$$
q_{i}=\frac{A_{i} k_{i}}{\mu}\left(\frac{\mathrm{d} p}{\mathrm{~d} x}-\lambda_{i}\right)
$$

where $q_{i}$ is flow in the capillary of the radius $r_{i}, A_{i}$ is crosssectional area of capillary radius $r_{i}, k_{i}$ is capillary permeability of radius $r_{i}, \mu$ is the viscosity, $(\mathrm{d} p / \mathrm{d} x)$ is the pressure gradient, and $\lambda_{i}$ is the threshold pressure gradient of capillary radius $r_{i}$.

Because the permeability of the small radius of the capillary is small, the threshold pressure gradient is large, so when the pressure gradient increases, the tiny capillaries start, the active permeability; namely, the increase of the straight slope of amplitude (that is the active permeability of the model varies with the rate of change of pressure gradient) should be gradually reduced. If a certain period of straight line slope increase amplitude is bigger, it is illustrated that, at a certain pressure gradient, the larger $A_{i}$ is, the more is the started capillary tube with the same or similar radius. If the capillary radius of model is according to the normal distribution and $A_{n}$ is 
TABLe 1: The fundamental physical parameters of core samples.

\begin{tabular}{lcccc}
\hline Core number & Diameter $(\mathrm{cm})$ & Length $(\mathrm{cm})$ & Gas logging permeability $\left(10^{-3} \mu \mathrm{m}^{2}\right)$ & Porosity $(\%)$ \\
\hline P11 & 2.516 & 5.242 & 1.97 & 13.43 \\
P12 & 2.508 & 5.268 & 2.39 & 13.55 \\
P13 & 2.516 & 5.594 & 3.45 & 13.47 \\
P14 & 2.506 & 5.164 & 8.93 & 14.99 \\
P15 & 2.504 & 6.242 & 9.96 & 14.43 \\
P16 & 2.504 & 5.798 & 1.81 & 14.96 \\
P17 & 2.512 & 5.20 & 26.1 & 16.27 \\
P18 & 2.502 & 5.934 & 28.91 & 17.83 \\
P19 & 2.504 & 6.046 & 31.07 & 18.12 \\
P20 & 2.504 & 5.438 & & \\
\hline
\end{tabular}

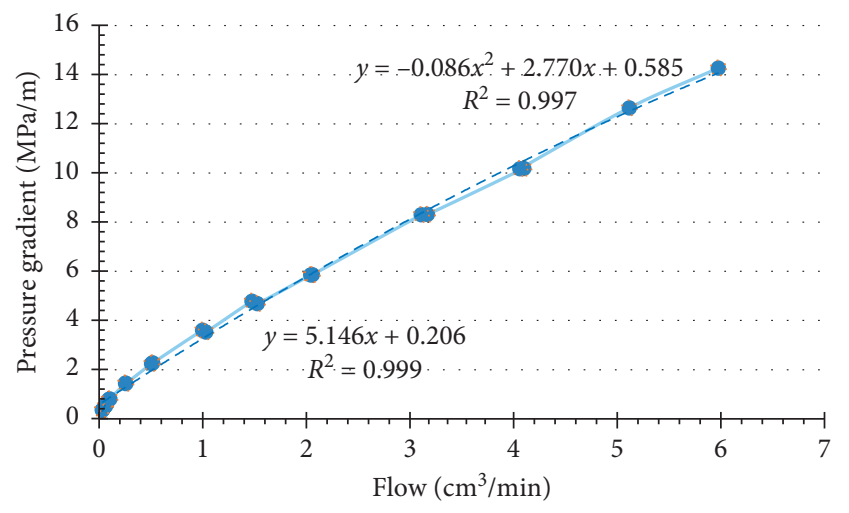

FIGURE 2: The relation diagram of the flow and the driving pressure gradient.

TABLE 2: The data of the flow and the driving pressure gradient.

\begin{tabular}{lc}
\hline Flow $Q\left(\mathrm{~cm}^{3} / \mathrm{min}\right)$ & The driving pressure gradient $\mathrm{d} p / \mathrm{d} x(\mathrm{MPa} / \mathrm{m})$ \\
\hline 0.030 & 0.35859 \\
0.030 & 0.36778 \\
0.060 & 0.51490 \\
0.061 & 0.53328 \\
0.094 & 0.78154 \\
0.102 & 0.80912 \\
0.255 & 1.45274 \\
0.257 & 1.43435 \\
0.515 & 2.28025 \\
0.506 & 2.24347 \\
0.998 & 3.60427 \\
1.029 & 3.53071 \\
1.476 & 4.78117 \\
1.526 & 4.68922 \\
2.042 & 5.84774 \\
2.058 & 5.86613 \\
3.109 & 8.29349 \\
3.167 & 8.31188 \\
4.098 & 10.18757 \\
4.060 & 10.16918 \\
5.116 & 12.65171 \\
5.976 & 14.26995 \\
\hline
\end{tabular}

according to the normal distribution, $f\left(k_{e}\right)$ should be first increased and then decreased. Both active permeability and threshold pressure gradient are in agreement, as shown in Figure 1.

$$
K_{a}=K_{e}-K_{e} \lambda_{e} \frac{\mathrm{d} x}{\mathrm{~d} p} .
$$

At the same time, the analysis above figure shows that the relationship between the apparent permeability and the reciprocal of the pressure gradient is a stable straight line when the pressure gradient is large; i.e., the reciprocal of the pressure gradient is small. The starting permeability of the straight line segment can be obtained by fitting the linear equation. The starting permeability $K_{e}$ is $K_{e}=16.725 \times 10^{-3} \mu \mathrm{m}^{2}$ and the quasistarting pressure gradient is $\lambda_{e}=1.44 \mathrm{MPa} / \mathrm{m}$.

3.1.3. The Threshold Pressure Gradient of Cores with Various Permeabilities. Based on a capillary bundle model, the analysis of the experimental results can be fitted according to the neighboring data points, because two experimental data points may result in jumps because of experimental errors, if the pressure gradients included in the selected four data points are larger, and different levels of pore throats open more, then resulting is inaccurate. Therefore, three experimental data points are selected for one fitting. The linear fitting of the flow and pressure gradient of the first three points (two measurements for each point) can be obtained. Thus, from the analysis, it can be seen that the whole lowpermeability seepage flow is nonlinear, but it is divided into some stages. The first stage is the strong nonlinear stage, the intermediate stage is quasilinear stage, and then the last stage is the linear stage.

The method can be used to obtain the threshold permeability and the threshold pressure gradient value fitted by the neighboring points, and then the relationship between the threshold permeability and the threshold pressure gradient value and the flow velocity can be made. Through $v=Q / A$, the experimental flow value is divided by the cross-sectional area of the core to convert the flow velocity, and the relationship between the starting permeability, the starting pressure gradient, and the flow velocity is obtained. The results are shown in Figures 3 and 4.

Figure 3 shows the quadratic function between the threshold permeability and the threshold pressure gradient with the flow rate. According to Figure 3, threshold permeability and threshold pressure gradient values at different pressure gradients (or flow rates) can be 


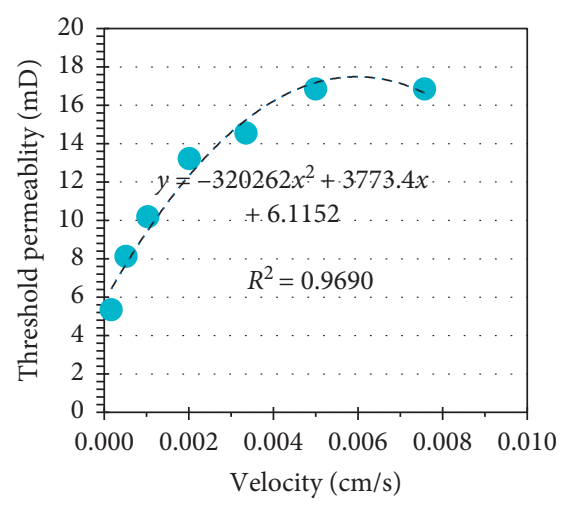

(a)

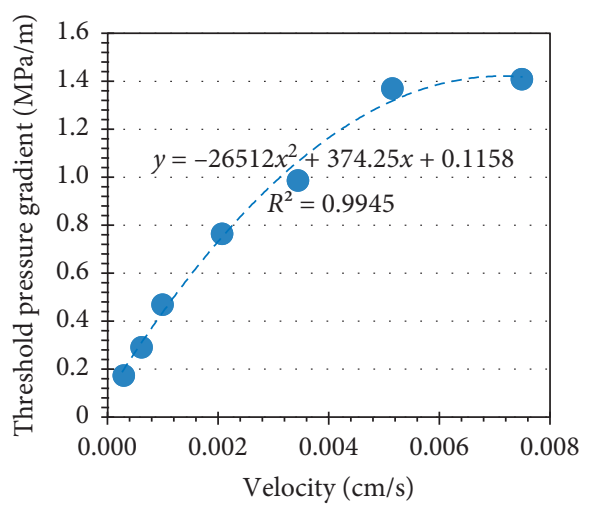

(b)

FIGURE 3: Fitted threshold pressure gradient changes and threshold permeability with velocity.

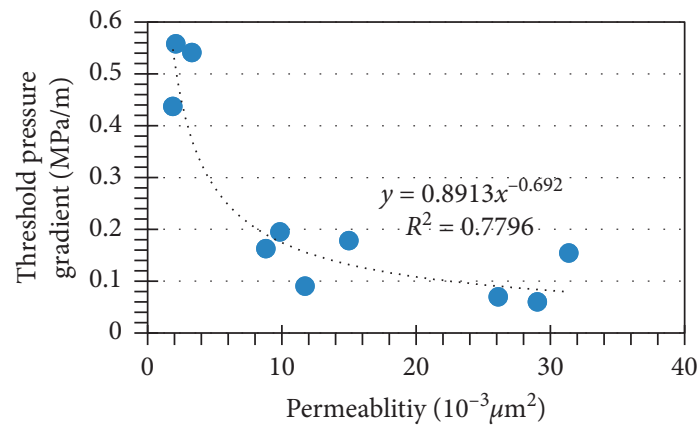

(a)

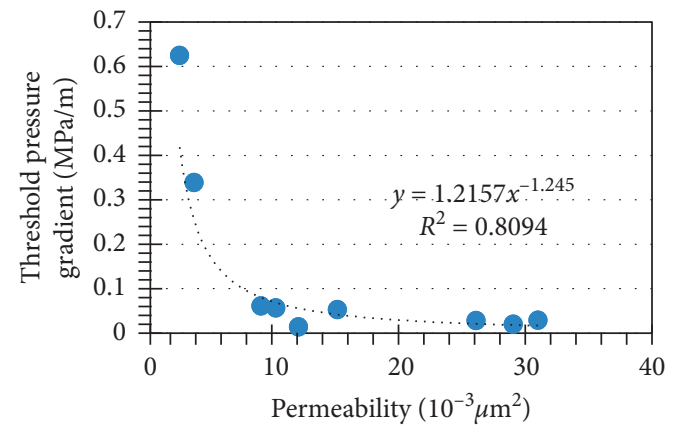

(b)

Figure 4: The calculated threshold pressure gradient. (a) Calculation using the first three data records. (b) Calculation using the new method.

interpolated. According to the first three points in Figure 3, the minimum threshold pressure gradient can be obtained by extrapolation. The data listed in Table 3 include the minimum threshold pressure gradient of each core, the threshold permeability and the threshold pressure gradient within the flow range of $0.1-0.6 \mathrm{~mL} / \mathrm{min}$, the threshold pressure gradient and threshold permeability obtained from the line segment without the influence of velocity sensitive effect, and the threshold pressure gradient.

In Figure 4(a), the threshold pressure gradient value is the minimum threshold pressure gradient fitted according to the matching pressure gradient of $0.03 \mathrm{~mL} / \mathrm{min}, 0.06 \mathrm{~mL}$ / $\mathrm{min}$, and $0.12 \mathrm{~mL} / \mathrm{min}$. In Figure $4(\mathrm{~b})$, the threshold pressure gradient value is the value when the flow rate is zero according to the threshold pressure gradient and flow rate curve showed.

The minimum threshold pressure gradient value does not decrease monotonically as the permeability increases, but rather fluctuates. The relationship between threshold pressure gradient and permeability in the range of $0.1-0.6 \mathrm{~mL} / \mathrm{min}$ can be fitted as

$$
\begin{aligned}
\lambda & =5.1813 k^{-0.87,}, \\
R^{2} & =0.8353,
\end{aligned}
$$

where $\lambda$ is the threshold pressure gradient, $\mathrm{MPa} / \mathrm{m} ; k$ is the permeability, $\mu \mathrm{m}^{2}$; and $R$ is the correlation coefficient.

The relationship between the threshold pressure gradient and the permeability obtained from the line slice without the influence of velocity sensitive effect can be fitted as

$$
\begin{aligned}
\lambda & =54.113 k^{-1.13}, \\
R^{2} & =0.9652,
\end{aligned}
$$

where $\lambda$ is the threshold pressure gradient, $\mathrm{MPa} / \mathrm{m} ; k$ is the permeability, $\mu \mathrm{m}^{2}$; and $R$ is the correlation coefficient.

It can be seen that the threshold pressure gradient has a better power function with the permeability. When permeability of core is more than $4 \mathrm{md}$, the threshold pressure gradient is small; when the permeability of core is less than $4 \mathrm{md}$, the threshold pressure gradient increases rapidly. The minimum threshold pressure gradient decreases as the permeability increases. The power function can better fit the variation of the data points. In Figure 4(b), the data points are smaller than those in Figure 4(a), and they can show the true minimum threshold pressure gradient better.

3.2. The Application of the Threshold Pressure Gradient in Oil Field Radial Flow. The condition of the threshold pressure gradient obtained in laboratory is one dimension, but the 
TABLE 3: The threshold pressure gradient of cores with various permeability.

\begin{tabular}{|c|c|c|c|c|c|c|}
\hline $\begin{array}{l}\text { Core } \\
\text { number }\end{array}$ & $\begin{array}{l}\text { Permeability, } \\
10^{-3} \mu \mathrm{m}^{2} \text { (md) }\end{array}$ & $\begin{array}{l}\text { Minimum } \\
\text { threshold pressure } \\
\text { gradient }(\mathrm{MPa} / \mathrm{m})\end{array}$ & $\begin{array}{l}0.1-0.6 \mathrm{~mL} / \mathrm{min} \text {, } \\
\text { threshold pressure } \\
\qquad(\mathrm{MPa} / \mathrm{m})\end{array}$ & $\begin{array}{l}0.1-0.6 \mathrm{~mL} / \mathrm{min} \text {, } \\
\text { start permeability } \\
(\mathrm{md})\end{array}$ & $\begin{array}{l}\text { Threshold pressure } \\
\text { gradient before the } \\
\text { velocity sensitive (MPa/ } \\
\mathrm{m} \text { ) }\end{array}$ & $\begin{array}{l}\text { Start permeability } \\
\text { before the } \\
\text { velocity sensitive } \\
\text { (md) }\end{array}$ \\
\hline P11 & 1.97 & 0.3205 & 3.504 & 0.440 & 22.246 & 0.420 \\
\hline P12 & 2.39 & 0.4209 & 1.711 & 0.561 & 19.572 & 0.697 \\
\hline P13 & 3.45 & 0.3383 & 2.559 & 0.546 & 18.515 & 1.154 \\
\hline P14 & 8.93 & 0.0626 & 0.576 & 0.166 & - & - \\
\hline P15 & 9.96 & 0.0495 & 1.138 & 0.197 & 3.843 & 4.216 \\
\hline P16 & 11.81 & 0.0161 & 0.450 & 0.092 & - & - \\
\hline P17 & 14.96 & 0.0521 & 0.324 & 0.185 & 1.816 & 6.661 \\
\hline P18 & 26.1 & 0.0299 & 0.213 & 0.073 & - & - \\
\hline P19 & 28.91 & 0.0198 & 0.290 & 0.062 & - & - \\
\hline $\mathrm{P} 20$ & 31.07 & 0.0276 & 0.484 & 0.160 & 1.179 & 16.582 \\
\hline
\end{tabular}

flow is radial flow in oilfield. From the foregoing analysis, it can be seen that the threshold pressure gradient changes with the flow velocity, while, in the radial flow, the velocity at different distances is different, so the threshold pressure gradient is different.

From the foregoing analysis, the relationship between the threshold pressure gradient and velocity can be fit as a quadratic function. The relationship between the start-up pressure gradient and the flow velocity is further analyzed and matched with the actual reservoir water injection, by the formula $v=(Q / 2 \pi r h)$; the change of flow velocity with the radius under different injection flow rate can be obtained. And its expression is shown as follows:

$$
\begin{aligned}
& \lambda=\alpha v^{2}+b v+c, \\
& \lambda=\alpha\left(\frac{Q}{2 \pi r h}\right)^{2}+b\left(\frac{Q}{2 \pi r h}\right)+c,
\end{aligned}
$$

where $\lambda$ is the threshold pressure gradient, $\mathrm{MPa} / \mathrm{m} ; \alpha, b$, and $c$ are the coefficients; $Q$ is the flow rate, $\mathrm{m}^{3} / \mathrm{s} ; r$ is the radius, $m$; and $h$ is the thickness, $m$.

According to equation (6), the threshold pressure gradient can be obtained at different distances from the center of the well. Integrating the radius $r$ can get the threshold pressure at different distances from the well center, as shown in the following formula:

$$
\begin{aligned}
P_{\lambda}= & \int_{r_{w}}^{r_{o}} \lambda \mathrm{d} r=\alpha\left(\frac{\mathrm{Q}}{2 \pi r h}\right)^{2}\left(\frac{1}{r_{w}}-\frac{1}{r_{o}}\right) \\
& +b\left(\frac{Q}{2 \pi r h}\right) \ln \left(\frac{r_{o}}{r_{w}}\right)+c\left(r_{o}-r_{w}\right),
\end{aligned}
$$

where $P_{\lambda}$ is the threshold pressure, $\mathrm{MPa} / \mathrm{m} ; r_{o}$ is the reservoir radius, $m ; r_{w}$ is the wellbore radius, $m$; $Q$ is the flow rate, $\mathrm{m}^{3} / \mathrm{s}$; and $h$ is the thickness, $m$.

Through analyzing the flow rates relations at different cores permeability, it shows that the permeability is approximate to linear with the flow rate (or velocity) before $0.002 \mathrm{~cm} / \mathrm{s}$, and after this flow rate, the slope of the permeability in linear relationship with the flow rate decreases. Therefore, the seepage equation can be divided into two sections before and after $0.002 \mathrm{~cm} / \mathrm{s}(1.728 \mathrm{~m} / \mathrm{d})$; that is, the relationship between core pressure gradient and velocity can be divided into low-speed and high-speed sections for quadratic function fitting.

Fitting the seepage equations (5) and (6) to the form of equation (8) yields

$$
\frac{\mathrm{d} p}{\mathrm{~d} x}=\alpha v^{2}+b v+c
$$

where $(\mathrm{d} p / \mathrm{d} x)$ is the driving pressure gradient, $\mathrm{MPa} / \mathrm{m} ; \alpha, b$, and $c$ are the coefficients; and $v$ is the velocity, $\mathrm{m} / \mathrm{d}$.

The seepage equation coefficients of cores with different gas logging permeability are listed in Table 4.

By fitting the equation, the relationship between the coefficient of the quadratic term of the equation and the permeability can be obtained, and the percolation equation at any permeability can be obtained.

The statistics of water injection data in the block: the results (Table 5) show that the injection intensity $Q / h$ is $0.3 \mathrm{~m}^{3} / \mathrm{d} / \mathrm{m} \sim 7.3 \mathrm{~m}^{3} / \mathrm{d} / \mathrm{m}$ mainly between $1.0 \mathrm{~m}^{3} / \mathrm{d} / \mathrm{m}$ $\sim 4.5 \mathrm{~m}^{3} / \mathrm{d} / \mathrm{m}$.

Figure 5 shows that the flow rate of injection intensity $Q /$ $h$ at $0.3 \mathrm{~m}^{3} / \mathrm{d} / \mathrm{m} \sim 7.3 \mathrm{~m}^{3} / \mathrm{d} / \mathrm{m}$ varies with the distance from the well center. According to the relationship between velocity and distance from the center of well, the relationship between starting pressure gradient and starting pressure and distance from the center of well can be obtained. As shown in Figure 6, the blue curve is the injection intensity $\mathrm{Q} / \mathrm{h}=1.0 \mathrm{~m}^{3} / \mathrm{d} / \mathrm{m}$, the red curve is the injection intensity $\mathrm{Q} / \mathrm{h}=4.5 \mathrm{~m}^{3} / \mathrm{d} / \mathrm{m}$, and the middle small figure is the local enlargement within $0.1 \sim 5 \mathrm{~m}$. The relationship between pressure gradient and pressure drop and distance from well center can be obtained.

As the method similar to equations (3) to (8), at the injection intensity of $Q / h=4.5 \mathrm{~m}^{3} / \mathrm{d} / \mathrm{m}$ and $\mathrm{Q} / \mathrm{h}=1.0 \mathrm{~m}^{3} / \mathrm{d} / \mathrm{m}$ conditions, take the threshold pressure values at the distance of $150 \mathrm{~m}$ away from the well center, which is obtained from cores with different permeability, and plot it. The result is shown in Figure 6.

With an injection rate of $Q / h=1.0 \mathrm{~m}^{3} / \mathrm{d} / \mathrm{m}$, the pressure drop at a certain distance can be obtained by integrating the distance by driving the pressure gradient. 
TABLE 4: The seepage equation fitting parameters for various permeability cores.

\begin{tabular}{|c|c|c|c|c|c|c|}
\hline \multirow{2}{*}{ Gas logging permeability $\times 10^{-3} \mu \mathrm{m}^{2}$} & \multicolumn{3}{|c|}{ Low flow, $<1.728 \mathrm{~m} / \mathrm{d}$} & \multicolumn{3}{|c|}{ High flow, $>1.728 \mathrm{~m} / \mathrm{d}$} \\
\hline & $a$ & $b$ & $c$ & $a_{2}$ & $b_{2}$ & $c_{2}$ \\
\hline 1.97 & 4401173 & 41495.94 & 0.270135 & 270974.2 & 29381.52 & 11.54866 \\
\hline 2.39 & 965176.1 & 23992.24 & 0.550795 & 299825.1 & 19745.3 & 6.815821 \\
\hline 3.45 & 2483235 & 20298.96 & 0.447727 & 106402.5 & 11171.91 & 11.77325 \\
\hline 8.93 & 690991.8 & 3742.243 & 0.12773 & 224498.1 & 3066.88 & 0.328465 \\
\hline 9.96 & 2120510 & 6538.008 & 0.078849 & 229873.3 & 3800.372 & 0.873352 \\
\hline 11.81 & 639844.8 & 6462.558 & 0.055449 & 205292.8 & 5856.899 & 0.220347 \\
\hline 14.96 & 546029.8 & 2904.804 & 0.125703 & 23705.57 & 1948.148 & 0.811546 \\
\hline 26.1 & 133718.5 & 1105.043 & 0.065861 & 0 & 775.07 & 0.2131 \\
\hline 28.91 & 416471.6 & 1405.897 & 0.035546 & 0 & 680.41 & 0.2896 \\
\hline 31.07 & 822100.9 & 2226.526 & 0.131521 & 6839.687 & 757.4748 & 1.009064 \\
\hline Fitting result & $4 E+06 k^{-0.724}$ & $79472 k^{-1.176}$ & $0.6633 k^{-0.722}$ & $4 E+6 k^{-1.034}$ & $68163 x^{-1.309}$ & $21.622 x^{-1.317}$ \\
\hline
\end{tabular}

TABLE 5: Variation of velocity with distance from well center at different injection flow rate.

\begin{tabular}{|c|c|c|c|c|c|c|}
\hline \multirow{2}{*}{$r(\mathrm{~m})$} & \multicolumn{6}{|c|}{$\mathrm{Q} / \mathrm{h}\left(\mathrm{m}^{3} / \mathrm{d} / \mathrm{m}\right)$} \\
\hline & 0.3 & 1 & 2 & 3 & 4.5 & 7.3 \\
\hline 0.1 & 0.478 & 1.592 & 3.185 & 4.777 & 7.166 & 11.624 \\
\hline 0.5 & 0.096 & 0.318 & 0.637 & 0.955 & 1.433 & 2.325 \\
\hline 1 & 0.048 & 0.159 & 0.318 & 0.478 & 0.717 & 1.162 \\
\hline 2 & 0.024 & 0.080 & 0.159 & 0.239 & 0.358 & 0.581 \\
\hline 5 & 0.010 & 0.032 & 0.064 & 0.096 & 0.143 & 0.232 \\
\hline 10 & 0.005 & 0.016 & 0.032 & 0.048 & 0.072 & 0.116 \\
\hline 50 & 0.001 & 0.003 & 0.006 & 0.010 & 0.014 & 0.023 \\
\hline 100 & 0.000 & 0.002 & 0.003 & 0.005 & 0.007 & 0.012 \\
\hline 150 & 0.000 & 0.001 & 0.002 & 0.003 & 0.005 & 0.008 \\
\hline
\end{tabular}

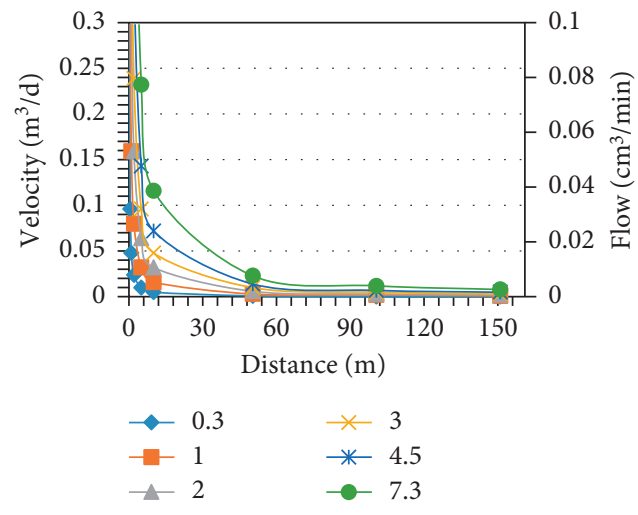

(a)

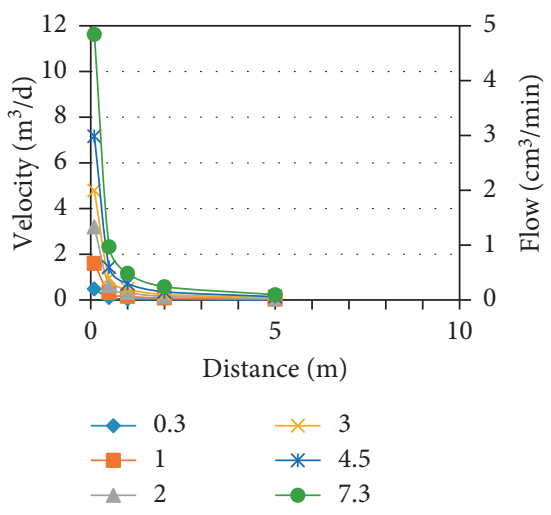

(b)

FIGURE 5: Variation of velocity with distance from well center. (a) $0.1 \sim 5 \mathrm{~m}$ distance from well center. (b) $0.1 \sim 150 \mathrm{~m}$ distance from well center.

The pressure drop values at a distance of $150 \mathrm{~m}$ from the center of the well with different permeability are plotted in Figure 6. The blue line is the direct calculation result of the pressure drop values calculated from the core driven pressure gradient and flow rate. The red line is the pressure drop values which are calculated from the value of the quadratic coefficient of the fitting percolation equation at different permeability. It can be seen from Figure 7 that the blue direct calculation value curve is very close to the power function curve fitted by the red fitting calculation value.

It is known from the following equation:

$$
P=P_{f}+P_{\lambda}=\int \frac{\mu}{A K_{e}} Q \mathrm{~d} x+\int \lambda_{e} \mathrm{~d} x
$$

where $P$ is the pressure, $\mathrm{MPa} ; P_{f}$ is the seepage pressure, $\mathrm{MPa}$; is the threshold pressure, $\mathrm{MPa} ; \mu$ is the viscosity, Pas; $A$ is the total cross-sectional area, $\mathrm{m}^{2} ; K_{e}$ is the threshold permeability, $\mu \mathrm{m}^{2} ; \lambda_{e}$ is the threshold pressure gradient, $\mathrm{MPa} / \mathrm{m}$; and $Q$ is the flow rate, $\mathrm{m}^{3} / \mathrm{s}$.

The total pressure drop $P$ is divided into two parts, which is the pressure drop $P_{f}$ caused by seepage and the threshold pressure $P_{\lambda}$. The small proportion of the threshold pressure to the total pressure drop indicates that the pressure drop 


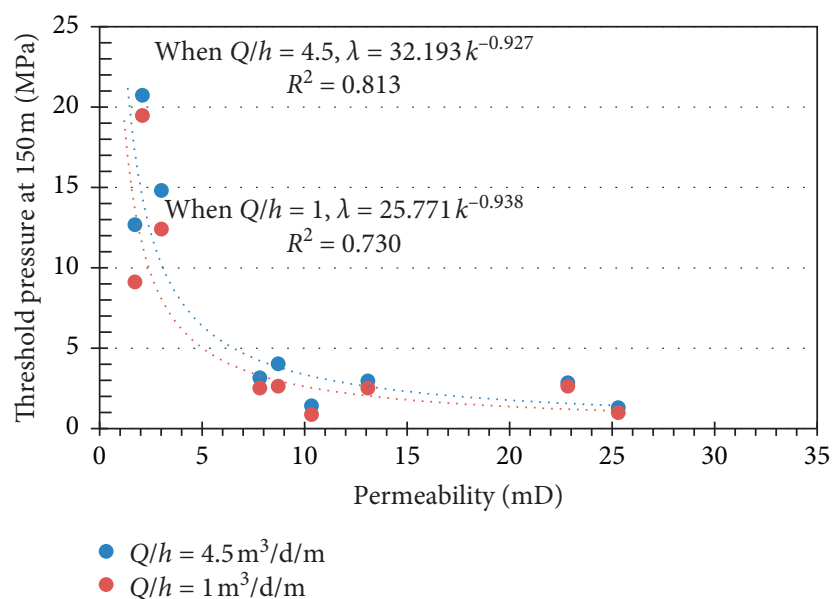

FIgURE 6: The threshold pressure at the distance of $150 \mathrm{~m}$ away from well center at different permeability.

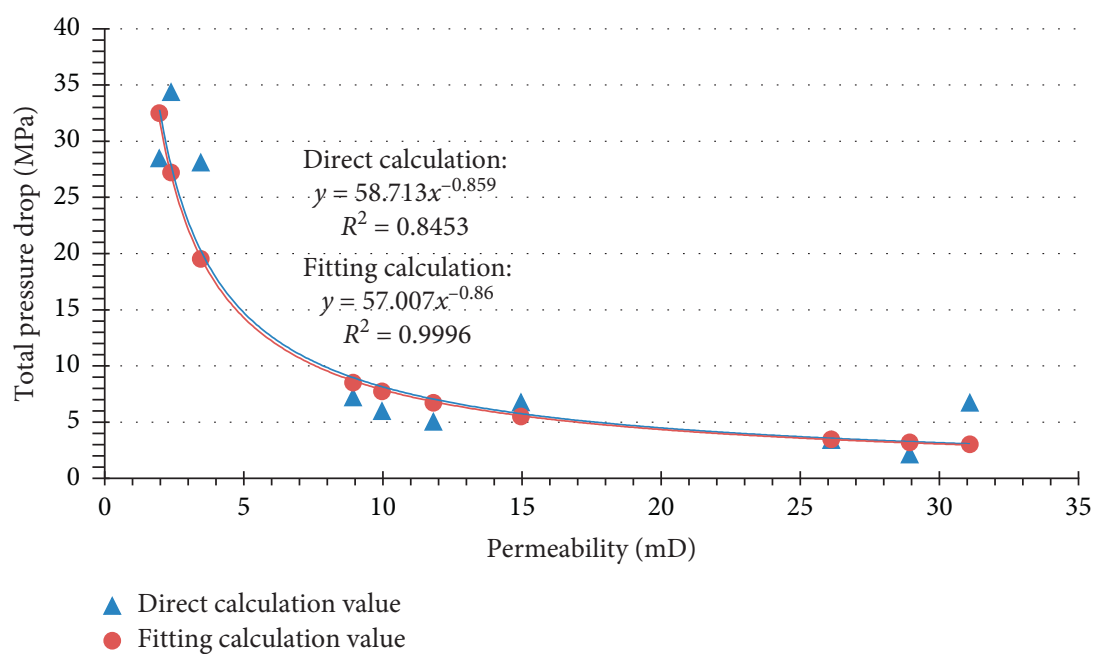

FIgURE 7: The total pressure drop at a distance of $150 \mathrm{~m}$ from the center of the well at different permeability.

used to overcome the threshold pressure is small which is advantageous. According to the fitting formulas $P_{\lambda}=25.771 k^{-0.938}, P=58.713 k^{-0.859}$ in when the injecting intensity is $Q / h=1.0 \mathrm{~m}^{3} / \mathrm{d} / \mathrm{m}$, we can get

$$
\frac{P_{\lambda}}{P}=0.4389 k^{-0.079} \text {, }
$$

where $P_{\lambda}$ is the threshold pressure, MPa; $P$ is the pressure, $\mathrm{MPa}$; and $k$ is the permeability, $\mu \mathrm{m}^{2}$.

From equation (10), it can be seen that as the permeability increases, the threshold pressure occupies a smaller proportion of the total pressure drop. The ratio of starting pressure to total pressure drop is about 0.5 , and the higher the permeability is, the lower the ratio is.

\section{Summary and Conclusions}

(1) In this paper, a capillary bundle model for calculating the threshold pressure gradient is established. It is believed that the core is composed of a large number of capillary bundles of different pore throats in parallel. In the process of percolation with increasing flow and pressure gradients, there are new capillaries continually starting in the pore throat. The flow rate increases, so the flow rate and pressure gradient curves are skewed toward the flow rate axis.

(2) During the displacement, the permeability gradually increases with the increase of flow rate. When the flow rate and pressure gradient are in a stable linear relationship, the apparent permeability and the reciprocal of the pressure gradient are in a straight line. Based on this, the threshold permeability and threshold pressure gradient can be fitted out.

(3) Through the analysis of experimental results, it shows the threshold pressure gradient and the threshold permeability increase with the increase of the flow rate and pressure gradient, and the increasing rate gradually becomes slower. If the flow rate is large, the threshold pressure gradient and the threshold permeability may be affected by the velocity sensitivity effect and decline. 
(4) According to the law of threshold pressure gradient with flow rate, the minimum threshold pressure gradient of each core, the threshold permeability and the threshold pressure gradient within the flow range of $0.1-0.6 \mathrm{~mL} / \mathrm{min}$, and the threshold permeability and the threshold pressure gradient at the straight line slice without the influence of velocity sensitive effect can be obtained. The minimum threshold pressure gradient and permeability, the threshold pressure gradient, and permeability all have a power function.

(5) The threshold pressure gradient and the flow velocity and the driving pressure gradient and the flow velocity are fitted according to the quadratic function, and the threshold pressure gradient, driving pressure gradient, threshold pressure, and driving pressure at different locations can be obtained. Fitting the threshold pressure and driving pressure at different permeability, it can be concluded that the threshold pressure and permeability, driving pressure, and permeability are in power function.

(6) Through the dynamic macro experiment and microcapillary bundle principle, the experiment can be divided into several sections for analysis, which can be more accurate. The minimum startup pressure gradient can not only guide the later development of the oilfield, but also enrich the theoretical study of non-Darcy low-velocity seepage. At the same time, the law of flow velocity and start-up pressure gradient indirectly proves the boundary layer theory of the generation mechanism of start-up pressure gradient and supports and guides the effective development of various development methods of low-permeability reservoir.

\section{Appendix}

\section{A. The Relationship of the Starting Permeability and the Quasistarting Pressure Gradient}

Because the largest radius capillary of the boundary layer thickness is small, the threshold pressure gradient is low. If the pressure gradient is tiny, then the fluid in the largest radius capillary firstly begins to flow. Therefore, the minimum threshold pressure gradient matches the maximum capillary radius. There is a certain threshold value in the pore radius, and the flow cannot start until the threshold value is reached, that is to say, the pore radius is related to the threshold pressure gradient. With the increase of pressure gradient, other capillary is in seepage flow.

$$
q_{a}=\sum_{i=1}^{n} \frac{A_{i} k_{i}}{\mu}\left(\frac{\mathrm{d} p}{\mathrm{~d} x}-\lambda_{i}\right) .
$$

For different radius of capillary tube threshold, with the linear fitting of the velocity and pressure gradient, the active permeability is formed:

$$
q_{a}=\frac{A k_{e}}{\mu}\left(\frac{\mathrm{d} p}{\mathrm{~d} x}-\lambda_{e}\right),
$$

where $q_{a}$ is the total flow of capillary bundle model; $A$ is total cross-sectional area of bundle of capillary tubes model; $k_{e}$ is the active permeability of the bundle of capillary tubes model; and $\lambda_{e}$ is the threshold pressure gradient of the bundle of capillary tubes model.

Combining equations (A.1) and (A.2), equations (A.3) and A.4) are obtained:

$$
\begin{aligned}
& k_{e}=\frac{1}{A} \sum_{i=1}^{n} A_{i} k_{i}, \\
& \lambda_{e}=\frac{\sum_{i=1}^{n} A_{i} k_{i} \lambda_{i}}{\sum_{i=1}^{n} A_{i} k_{i}} .
\end{aligned}
$$

Types (A.3) and (A.4) show that the active permeability is the permeability weighted superposition that has been started in the threshold of the different radius capillary. With the increase of pressure gradient, small capillary is opened; the threshold pressure gradient and the active permeability gradually increase. The change of the active permeability with pressure gradient is as follows:

$$
\begin{aligned}
& f\left(k_{e}\right)=\frac{\sum_{i=1}^{n} A_{i} k_{i}-\sum_{i=1}^{n} A_{i} k_{i} \lambda_{i}}{A \Delta(\mathrm{d} p / \mathrm{d} x)}=\frac{A_{n} k_{n}}{A \Delta(\mathrm{d} p / \mathrm{d} x)}, \\
& f\left(\lambda_{e}\right)=\frac{1}{\Delta(\mathrm{d} p / \mathrm{d} x)}\left(\frac{\sum_{i=1}^{n} A_{i} k_{i} \lambda_{i}}{\sum_{i=1}^{n} A_{i} k_{i}}-\frac{\sum_{i=1}^{n-1} A_{i} k_{i} \lambda_{i}}{\sum_{i=1}^{n-1} A_{i} k_{i}}\right), \\
& f\left(\lambda_{e}\right)<\frac{A_{n} k_{n} \lambda_{n}}{\Delta(\mathrm{d} p / \mathrm{d} x) \sum_{i=1}^{n} A_{i} k_{i}} .
\end{aligned}
$$

\section{Data Availability}

The experiment data used to support the findings of this study are available from the corresponding author upon request.

\section{Conflicts of Interest}

The authors declare that they have no conflicts of interest.

\section{References}

[1] Y. Huang, Flow Mechanics for Low Permeability Reservoir, Petroleum Industry Press, Beijing, China, 1998

[2] C. Lv, J. Wang, and Z. Sun, “An experimental study on starting pressure gradient of fluids flow in low permeability sandstone porous media," Petroleum Exploration and Development, vol. 29, no. 2, pp. 86-89, 2002.

[3] Q. Yan, Q. He, W. Ligang et al., "An experimental study on one phase fluid flow in low permeability reservoir," Journal of Xi'an Shiyou University, vol. 5, no. 2, pp. 1-6, 1990.

[4] L. K. Thomas, D. L. Katz, and M. R. Tek, "Threshold pressure phenomena in porous media," Society of Petroleum Engineers Journal, vol. 8, no. 2, 1968.

[5] H. Han, L. Cheng, M. Zhang et al., "Physical simulation and numerical simulation of ultra-low permeability reservoir in 
consideration of starting pressure gradient," Journal of the University of Petroleum, vol. 28, no. 6, pp. 49-53, 2004.

[6] V. A. Baikov, A. Y. Davletbaev, and D. S. Ivaschenko, "Nondarcy flow numerical simulation and pressure/rate transient analysis for ultra-low permeable reservoirs," in Proceedings of the SPE Russian Oil and Gas Exploration \& Production Technical Conference and Exhibition, Moscow, Russia, October 2014.

[7] J. Liu, X. Zhao, X. Liao et al., "New method to confirm the starting pressure gradient for low permeability reservoir," Science Technology and Engineering, vol. 12, no. 32, pp. 8518-8520, 2012.

[8] A. Li, M. Liu, S. Zhang et al., "Experimental study on the percolation characteristic of extra low-permeability reservoir," Journal of Xi'an Shiyou University, vol. 23, no. 2, pp. 35-39, 2008.

[9] J. Xu, L. Cheng, Y. Zhou et al., "A new method for calculating kickoff pressure gradient," Petroleum Exploration and Development, vol. 34, no. 5, pp. 594-597, 2007.

[10] L. Sun, Wufan, W. Zhao et al., "The study and application of reservoir start-up pressure," Fault Block Oil \& Gas Field, vol. 5, no. 5, pp. 30-33, 1998.

[11] Q. Wang, H. Tang, D. Lv et al., "An experimental study on threshold pressure gradient in low permeability reservoir," Petroleum Geology and Recovery Efficiency, vol. 18, no. 1, pp. 97-100, 2011.

[12] M. Kutiĺek, "Non-darcian flow of water in soils-laminar region: a review," Developments in Oil Science, vol. 2, pp. 327-340, 1972.

[13] H. Fei, L. Cheng, C. Li et al., "Study on threshold pressure gradient in ultra-low permeability reservoir," Journal of Southwest Petroleum Institute, vol. 28, no. 6, pp. 29-32, 2006.

[14] J. Yan, Y. Qi, and X. Liu, "Analysis on starting pressure gradient of oil phase at irreducible water in low permeability oil reservoirs," Fault Block Oil \& Gas Field, vol. 21, no. 3, pp. 344-347, 2014.

[15] W. Xiong, Q. Lei, X. Liu et al., "Pseudo threshold pressure gradient to flow for low permeability reservoirs," Petroleum Exploration and Development, vol. 36, no. 2, pp. 232-236, 2009. 